\title{
CHARACTERIZATION OF PARABOLIC HARDY SPACES BY LITTLEWOOD-PALEY FUNCTIONS
}

\author{
SHUICHI SATO
}

\begin{abstract}
We consider Littlewood-Paley functions associated with non-isotropic dilations. We prove that they can be used to characterize the parabolic Hardy spaces of Calderón-Torchinsky.
\end{abstract}

\section{INTRODUCTION}

Let $P$ be an $n \times n$ real matrix such that

$$
\langle P x, x\rangle \geq\langle x, x\rangle \text { for all } x \in \mathbb{R}^{n},
$$

where $\langle x, y\rangle=x_{1} y_{1}+\cdots+x_{n} y_{n}$ is the inner product in $\mathbb{R}^{n}$. Let $\gamma=\operatorname{trace} P$. Define a dilation group $\left\{A_{t}\right\}_{t>0}$ on $\mathbb{R}^{n}$ by $A_{t}=t^{P}=\exp ((\log t) P)$. It is known that $\left|A_{t} x\right|$ is strictly increasing as a function of $t$ on $\mathbb{R}_{+}=(0, \infty)$ for $x \neq 0$, where $|x|=\langle x, x\rangle^{1 / 2}$. Define a norm function $\rho(x)$ to be the unique positive real number $t$ such that $\left|A_{t^{-1}} x\right|=1$ when $x \neq 0$ and $\rho(0)=0$. Then $\rho\left(A_{t} x\right)=t \rho(x), t>0$, $x \in \mathbb{R}^{n}, \rho \in C^{\infty}\left(\mathbb{R}^{n} \backslash\{0\}\right)$ and the following results are known (see [4, 6]):

(P.1) $\rho(x+y) \leq \rho(x)+\rho(y)$;

(P.2) $\rho(x) \leq 1$ if and only if $|x| \leq 1$;

(P.3) $|x| \leq \rho(x)$ if $|x| \leq 1$;

(P.4) $|x| \geq \rho(x)$ if $|x| \geq 1$;

(P.5) $\left|A_{t} x\right| \geq t|x|$ if $t \geq 1$ for all $x \in \mathbb{R}^{n}$;

(P.6) $\left|A_{t} x\right| \leq t|x|$ if $0<t \leq 1$ for all $x \in \mathbb{R}^{n}$.

Similarly, we can consider a norm function $\rho^{*}(x)$ associated with the dilation group $\left\{A_{t}^{*}\right\}_{t>0}$, where $A_{t}^{*}$ denotes the adjoint of $A_{t}$. We have properties analogous to those for $\rho(x), A_{t}$ above.

Let

$$
g_{\varphi}(f)(x)=\left(\int_{0}^{\infty}\left|f * \varphi_{t}(x)\right|^{2} \frac{d t}{t}\right)^{1 / 2}
$$

be the Littlewood-Paley function on $\mathbb{R}^{n}$, where $\varphi_{t}(x)=t^{-\gamma} \varphi\left(A_{t}^{-1} x\right)$ and $\varphi$ is a function in $L^{1}\left(\mathbb{R}^{n}\right)$ such that

$$
\int_{\mathbb{R}^{n}} \varphi(x) d x=0 .
$$

For $L^{p}$ boundedness of $g_{\varphi}, 1<p<\infty$, see [1, 15.

2010 Mathematics Subject Classification. Primary 42B25; Secondary 42B30.

Key Words and Phrases. Littlewood-Paley functions, parabolic Hardy spaces.

The author is partly supported by Grant-in-Aid for Scientific Research (C) No. 25400130, Japan Society for the Promotion of Science. 
We say that a tempered distribution $f$ belongs to the parabolic $H^{p}$ if $\|f\|_{H^{p}}=$ $\left\|f^{*}\right\|_{p}<\infty$, where $f^{*}(x)=\sup _{t>0}\left|\Phi_{t} * f(x)\right|$ and $\left\|f^{*}\right\|_{p}=\left\|f^{*}\right\|_{L^{p}}$, with $\Phi \in \mathcal{S}\left(\mathbb{R}^{n}\right)$ satisfying $\int \Phi(x) d x=1, \operatorname{supp}(\Phi) \subset\{|x| \leq 1\}$ (see [4, 5], 8]). We have denoted by $\mathcal{S}\left(\mathbb{R}^{n}\right)$ the Schwartz class of rapidly decreasing smooth functions on $\mathbb{R}^{n}$.

Let $\varphi \in L^{1}\left(\mathbb{R}^{n}\right)$. We consider the non-degeneracy condition:

$$
\sup _{t>0}\left|\hat{\varphi}\left(A_{t}^{*} \xi\right)\right|>0 \quad \text { for all } \xi \neq 0,
$$

where the Fourier transform is defined as

$$
\hat{f}(\xi)=\mathcal{F}(f)(\xi)=\int_{\mathbb{R}^{n}} f(x) e^{-2 \pi i\langle x, \xi\rangle} d x .
$$

In this note we shall prove the following.

Theorem 1.1. Let $\varphi$ be a function in $\mathcal{S}\left(\mathbb{R}^{n}\right)$ satisfying (1.2) and (1.3). Let $0<$ $p \leq 1$. Then if $f \in H^{p}$, we have

$$
c_{1}\|f\|_{H^{p}} \leq\left\|g_{\varphi}(f)\right\|_{p} \leq c_{2}\|f\|_{H^{p}}
$$

with some positive constants $c_{1}, c_{2}$ independent of $f$.

We recall some related results when $P=E$ (the identity matrix) and $g_{\varphi}$ is defined by $\varphi_{t}(x)=t^{-n} \varphi\left(t^{-1} x\right)$ in (1.1). Then Theorem 1.1 is known (see [19] and also [11] for some background materials). Let $Q(x)=[(\partial / \partial t) P(x, t)]_{t=1}$, where

$$
P(x, t)=c_{n} \frac{t}{\left(|x|^{2}+t^{2}\right)^{(n+1) / 2}}, \quad c_{n}=\frac{\Gamma((n+1) / 2)}{\pi^{(n+1) / 2}},
$$

is the Poisson kernel associated with the upper half space $\mathbb{R}^{n} \times(0, \infty)$ (see [17, Chap. I]). We note that $\hat{Q}(\xi)=-2 \pi|\xi| e^{-2 \pi|\xi|}$. Then it is also known that

$$
c_{1}\|f\|_{H^{p}} \leq\left\|g_{Q}(f)\right\|_{p} \leq c_{2}\|f\|_{H^{p}}
$$

for $f \in H^{p}\left(\mathbb{R}^{n}\right), 0<p<\infty$, with positive constants $c_{1}, c_{2}$ (see [8] and also [19]). In [9, Chap. 7] we can find a relation between Hardy spaces and Littlewood-Paley functions associated with the heat kernel on homogeneous groups.

Uchiyama [19] gave a proof of the first inequality of (1.4) for $0<p \leq 1$ by methods of real analysis without the use of special properties of the Poisson kernel such as harmonicity, a semigroup property. Applying a similar argument, [19] proved the first inequality of the conclusion of Theorem 1.1 (when $P=E$ ) for $0<p \leq 1$ :

$$
\|f\|_{H^{p}} \leq c\left\|g_{\varphi}(f)\right\|_{p} .
$$

For a function $F$ on $\mathbb{R}^{n}$ and positive real numbers $N, R$, define the Peetre maximal function $F_{N, R}^{* *}$ by

$$
F_{N, R}^{* *}(x)=\sup _{y \in \mathbb{R}^{n}} \frac{|F(x-y)|}{(1+R|y|)^{N}}
$$

(see [13]). Then, it is known that the maximal function $F_{N, R}^{* *}$ can be used along with well-known arguments to prove (1.5) for $0<p \leq 1$ when $\varphi \in \mathcal{S}\left(\mathbb{R}^{n}\right)$ satisfies a non-degeneracy condition and the condition $\operatorname{supp}(\hat{\varphi}) \subset\left\{a_{1} \leq|\xi| \leq a_{2}\right\}$ for $a_{1}, a_{2}>0$.

In [16], (1.5) was proved for $f$ in a dense subspace of $H^{p}\left(\mathbb{R}^{n}\right)$ and for $\varphi$ in a class of functions including $Q$ and a general $\varphi \in \mathcal{S}\left(\mathbb{R}^{n}\right)$, without the restriction on $\operatorname{supp}(\hat{\varphi})$ above, with (1.2) and (1.3) by applying a vector valued inequality related to the Littlewood-Paley theory. The proof of the vector valued inequality is based 
on an application of the maximal function $F_{N, R}^{* *}$. This proof of (1.5) was discovered by [16.

The purpose of this note is to generalize the methods of [16] to the case of the parabolic Hardy spaces and get the characterization of the parabolic Hardy spaces in terms of Littlewood-Paley functions (Theorem 1.1).

In Section 2, we shall prove an analogue of the vector valued inequality in 16 for the general dilation group $\left\{A_{t}\right\}$ (Theorem 2.4). We shall consider $g_{\varphi}$ for $\varphi$ in a class of functions which includes those $\varphi$ considered in Theorem 1.1 and prove (1.5) in the case of the parabolic $H^{p}$ for such $\varphi$ and for $f$ in a dense subspace of $H^{p}$ as an application of Theorem 2.4 (Corollary 3.1). Theorem 2.4 will be stated more generally than needed for the proof of Corollary 3.1 as weighted vector valued inequalities.

In Section 3, a proof of Corollary 3.1 will be given by applying Theorem 2.4 and an atomic decomposition for Hardy spaces. Also, Theorem 1.1 will be derived from Corollary 3.1 .

\section{Weighted VECtor VALUED INEQUALITIES With NON-ISOTROPIC DILATIONS}

We need a partition of unity on $\mathbb{R}^{n} \backslash\{0\}$ associated with $\varphi$ satisfying (1.3).

Lemma 2.1. Suppose that $\varphi$ is a function in $L^{1}\left(\mathbb{R}^{n}\right)$ satisfying (1.3). We assume that $\hat{\varphi} \in C^{\infty}\left(\mathbb{R}^{n} \backslash\{0\}\right)$. Then, there exist $b_{0} \in(0,1)$ and $r_{1}, r_{2}>0, r_{1}<r_{2}$, such that for any $b \in\left[b_{0}, 1\right)$ there exists a function $\eta$ with the following properties:

(1) $\eta \in C^{\infty}\left(\mathbb{R}^{n}\right)$;

(2) $\hat{\eta} \in C^{\infty}\left(\mathbb{R}^{n}\right)$ and $\operatorname{supp} \hat{\eta} \subset\left\{r_{1}<\rho^{*}(\xi)<r_{2}\right\}$;

(3) $\sum_{j=-\infty}^{\infty} \hat{\varphi}\left(A_{b^{j}}^{*} \xi\right) \hat{\eta}\left(A_{b^{j}}^{*} \xi\right)=1 \quad$ for $\xi \in \mathbb{R}^{n} \backslash\{0\}$.

When $P$ is the identity matrix, this is in [18, Chap. V]; see also [4].

Proof of Lemma 2.1. Let $S^{n-1}=\{\xi:|\xi|=1\}$. Since $\hat{\varphi}$ is continuous, by a compactness argument we can find a finite family $\left\{I_{h}\right\}_{h=1}^{L}$ of compact intervals in $(0, \infty)$ such that

$$
\inf _{\xi \in S^{n-1}} \max _{1 \leq h \leq L} \inf _{t \in I_{h}}\left|\hat{\varphi}\left(A_{t}^{*} \xi\right)\right|^{2} \geq c
$$

with a positive constant $c$.

We observe that there exists $b_{0} \in(0,1)$ such that if $b \in\left[b_{0}, 1\right), t>0$ and $1 \leq h \leq L$, then we have $b^{j} t \in I_{h}$ for some $j \in \mathbb{Z}$ (the set of integers). This can be seen by taking $b_{0}=\max _{1 \leq h \leq L}\left(a_{h} / b_{h}\right)$, where $I_{h}=\left[a_{h}, b_{h}\right]$.

Consider an interval $[m, H]$ in $(0, \infty)$ such that $\cup_{h=1}^{L} I_{h} \subset[m, H]$ and choose $\theta \in C_{0}^{\infty}(\mathbb{R})$ such that $\theta=1$ on $[m, H], \operatorname{supp} \theta \subset[m / 2,2 H], \theta \geq 0$. Define

$$
\Psi(\xi)=\sum_{j=-\infty}^{\infty} \theta\left(b^{j} \rho^{*}(\xi)\right)\left|\hat{\varphi}\left(A_{b^{j}}^{*} \xi\right)\right|^{2} .
$$

Then $\Psi(\xi) \geq c>0$ for $\xi \neq 0$. Note that $\Psi\left(A_{b^{k}}^{*} \xi\right)=\Psi(\xi)$ for $k \in \mathbb{Z}$. Let

$$
\hat{\eta}(\xi)=\theta\left(\rho^{*}(\xi)\right) \overline{\hat{\varphi}(\xi)} \Psi(\xi)^{-1} \quad \text { for } \xi \neq 0
$$

and $\hat{\eta}(0)=0$, where $\overline{\hat{\varphi}(\xi)}$ denotes the complex conjugate. Then, $\eta$ satisfies all the properties stated in the lemma. This completes the proof.

To state our results, we introduce a class of functions. 
Definition 2.2. Let $\varphi$ be as in Lemma 2.1. Then, we say $\varphi \in B$ if the following conditions are satisfied:

$$
\begin{gathered}
\varphi \in C^{1}\left(\mathbb{R}^{n}\right), \quad \partial_{k} \varphi \in L^{1}\left(\mathbb{R}^{n}\right), \quad 1 \leq k \leq n \\
|\hat{\varphi}(\xi)| \leq C|\xi|^{\epsilon} \quad \text { for some } \epsilon>0 \\
\left|\partial_{\xi}^{\alpha} \hat{\varphi}(\xi)\right| \leq C_{\alpha, \tau}|\xi|^{-\tau} \quad \text { outside a neighborhood of the origin, }
\end{gathered}
$$

for all $\alpha$ and $\tau>0$ with a constant $C_{\alpha, \tau}$, where $\alpha=\left(\alpha_{1}, \ldots, \alpha_{n}\right)$ is a multi-index, $\alpha_{j} \in \mathbb{Z}, \alpha_{j} \geq 0,|\alpha|=\alpha_{1}+\cdots+\alpha_{n}$ and $\partial_{\xi}^{\alpha}=\partial_{\xi_{1}}^{\alpha_{1}} \ldots \partial_{\xi_{n}}^{\alpha_{n}}, \partial_{\xi_{k}}=\partial / \partial_{\xi_{k}}$.

If $\psi \in \mathcal{S}\left(\mathbb{R}^{n}\right)$ with (1.2) and (1.3), then clearly $\psi \in B$.

Let $\varphi \in B$. Then (2.1) implies that

$$
\mathcal{F}\left(\partial_{k} \varphi\right)(\xi)=\Xi_{k}(\xi) \hat{\varphi}(\xi), \quad 1 \leq k \leq n,
$$

where $\Xi_{k}(\xi)=2 \pi i \xi_{k}$ and $\partial_{k}=\partial_{x_{k}}$. Let $b \in\left[b_{0}, 1\right)$ and $\eta$ be as in Lemma 2.1. Define

$$
\hat{\zeta}(\xi)=1-\sum_{j \geq 0} \hat{\varphi}\left(A_{b^{j}}^{*} \xi\right) \hat{\eta}\left(A_{b^{j}}^{*} \xi\right) .
$$

Then $\operatorname{supp}(\hat{\zeta}) \subset\left\{\rho^{*}(\xi) \leq r_{2}\right\}, \hat{\zeta}=1$ in $\left\{\rho^{*}(\xi)<r_{1}\right\}$ and by (2.4) we have

$$
\begin{aligned}
\mathcal{F}\left(\partial_{k} \varphi\right)(\xi) & =\sum_{j \geq 0} \mathcal{F}\left(\partial_{k} \varphi\right)(\xi) \hat{\varphi}\left(A_{b^{j}}^{*} \xi\right) \hat{\eta}\left(A_{b^{j}}^{*} \xi\right)+\hat{\zeta}(\xi) \mathcal{F}\left(\partial_{k} \varphi\right)(\xi) \\
& =\sum_{j \geq 0} \hat{\varphi}\left(A_{b^{j}}^{*} \xi\right) \mathcal{F}\left(\alpha_{(k)}^{\left(b^{j}\right)}\right)\left(A_{b^{j}}^{*} \xi\right)+\hat{\varphi}(\xi) \mathcal{F}\left(\beta_{(k)}\right)(\xi),
\end{aligned}
$$

where $\alpha_{(k)}^{\left(b^{j}\right)}(x)=\left(\partial_{k} \varphi\right)_{b^{-j}} * \eta(x)$ and $\mathcal{F}\left(\beta_{(k)}\right)(\xi)=\hat{\zeta}(\xi) \Xi_{k}(\xi)$.

Thus we have

$$
\left|F\left(\partial_{k} \varphi, f\right)(x, t)\right| \leq \sum_{j \geq 0}\left|F\left(\alpha_{(k)}^{\left(b^{j}\right)} * \varphi, f\right)\left(x, b^{j} t\right)\right|+\left|F\left(\beta_{(k)} * \varphi, f\right)(x, t)\right|,
$$

where $f \in \mathcal{S}\left(\mathbb{R}^{n}\right)$ and $F(\psi, f)(x, t)=f * \psi_{t}(x)$. We also write $F(\psi, f)(x, t)=$ $F_{\psi}(x, t)$ when $f$ is fixed.

Let

$$
C_{0}\left(\partial_{k} \varphi, t, L, x\right)=(1+\rho(x))^{L}\left|\int \mathcal{F}\left(\partial_{k} \varphi\right)\left(A_{t^{-1}}^{*} \xi\right) \hat{\eta}(\xi) e^{2 \pi i\langle x, \xi\rangle} d \xi\right|,
$$

with $t>0, L \geq 0$. Then

$$
\left|\alpha_{(k) s}^{\left(b^{j}\right)}(x)\right|=C_{0}\left(\partial_{k} \varphi, b^{j}, L, A_{s}^{-1} x\right) s^{-\gamma}(1+\rho(x) / s)^{-L}
$$

for $j \in \mathbb{Z}$. Similarly,

$$
\left|\beta_{(k) s}(x)\right|=D\left(\Xi_{k}, L, A_{s}^{-1} x\right) s^{-\gamma}(1+\rho(x) / s)^{-L},
$$

with

$$
D\left(\Xi_{k}, L, x\right)=(1+\rho(x))^{L}\left|\int \hat{\zeta}(\xi) \Xi_{k}(\xi) e^{2 \pi i\langle x, \xi\rangle} d \xi\right| .
$$


Put $C\left(\partial_{k} \varphi, j, L, x\right)=C_{0}\left(\partial_{k} \varphi, b^{j}, L, x\right), j \in \mathbb{Z}$. Define

$$
\begin{gathered}
C\left(\partial_{k} \varphi, j, L\right)=\int_{\mathbb{R}^{n}} C\left(\partial_{k} \varphi, j, L, x\right) d x, \quad j \in \mathbb{Z}, \\
D\left(\Xi_{k}, L\right)=\int_{\mathbb{R}^{n}} D\left(\Xi_{k}, L, x\right) d x .
\end{gathered}
$$

We can consider $C(\psi, j, L)$ for other $\psi \in L^{1}\left(\mathbb{R}^{n}\right)$ by (2.6) and (2.8) with $\psi$ in place of $\partial_{k} \varphi$. We also write $C(\psi, j, L)=C_{\varphi}(\psi, j, L), D\left(\Xi_{k}, L\right)=D_{\varphi}\left(\Xi_{k}, L\right)$ to indicate that these quantities are based on $\varphi$. We have $D\left(\Xi_{k}, L\right), C\left(\partial_{k} \varphi, j, L\right)<\infty$ for all $j, L$, which can be seen from Lemma 2.9 below.

Let

$$
F_{N, R}^{* *}(x)=\sup _{y \in \mathbb{R}^{n}} \frac{|F(x-y)|}{(1+R \rho(y))^{N}}
$$

for a function $F$ on $\mathbb{R}^{n}$ and positive real numbers $N, R$. We need the following result in proving Theorem 2.4 below.

Lemma 2.3. Let $\varphi \in B, b \in\left[b_{0}, 1\right), N>0$. Then we have

$$
\begin{array}{r}
F\left(\partial_{k} \varphi, f\right)(\cdot, t)_{N, t^{-1}}^{* *}(x) \leq C \sum_{j \geq 0} C\left(\partial_{k} \varphi, j, N\right) b^{-j N} F(\varphi, f)\left(\cdot, b^{j} t\right)_{N,\left(b^{j} t\right)^{-1}}^{* *}(x) \\
+C D\left(\Xi_{k}, N\right) F(\varphi, f)(\cdot, t)_{N, t^{-1}}^{* *}(x)
\end{array}
$$

for $1 \leq k \leq n$, where $f \in \mathcal{S}\left(\mathbb{R}^{n}\right)$.

Proof. By (2.5) we have

$$
\begin{aligned}
& \left|F_{\partial_{k} \varphi}(z, t)\right| \\
& \leq C \sum_{j \geq 0} \int\left|F_{\varphi}\left(y, b^{j} t\right)\right|\left(1+\frac{\rho(z-y)}{b^{j} t}\right)^{-N} C\left(\partial_{k} \varphi, j, N, A_{b^{j} t}^{-1}(z-y)\right)\left(b^{j} t\right)^{-\gamma} d y \\
& +C \int\left|F_{\varphi}(y, t)\right|\left(1+\frac{\rho(z-y)}{t}\right)^{-N} D\left(\Xi_{k}, N, A_{t^{-1}}(z-y)\right) t^{-\gamma} d y .
\end{aligned}
$$

Multiplying both sides of the inequality by $(1+\rho(x-z) / t)^{-N}$ and noting that

$$
\left(1+\frac{\rho(z-y)}{b^{j} t}\right)^{-N}\left(1+\frac{\rho(x-z)}{t}\right)^{-N} \leq C_{A, N} b^{-N j}\left(1+\frac{\rho(x-y)}{b^{j} t}\right)^{-N}
$$


for any $x, y, z \in \mathbb{R}^{n}$ and $t>0$ when $b^{j} \leq A$, we have

$$
\begin{aligned}
& \left|F_{\partial_{k} \varphi}(z, t)\right|(1+\rho(x-z) / t)^{-N} \\
& \leq C \sum_{j \geq 0} b^{-N j} \int\left|F_{\varphi}\left(y, b^{j} t\right)\right|\left(1+\frac{\rho(x-y)}{b^{j} t}\right)^{-N} C\left(\partial_{k} \varphi, j, N, A_{b^{j} t}^{-1}(z-y)\right)\left(b^{j} t\right)^{-\gamma} d y \\
& \quad+C \int\left|F_{\varphi}(y, t)\right|\left(1+\frac{\rho(x-y)}{t}\right)^{-N} D\left(\Xi_{k}, N, A_{t}^{-1}(z-y)\right) t^{-\gamma} d y \\
& \leq C \sum_{j \geq 0} b^{-N j} F_{\varphi}\left(\cdot, b^{j} t\right)_{N,\left(b^{j} t\right)^{-1}}^{* *}(x) \int C\left(\partial_{k} \varphi, j, N, A_{b^{j} t}^{-1}(z-y)\right)\left(b^{j} t\right)^{-\gamma} d y \\
& \quad+C F_{\varphi}(\cdot, t)_{N, t^{-1}}^{* *}(x) \int D\left(\Xi_{k}, N, A_{t}^{-1}(z-y)\right) t^{-\gamma} d y \\
& \leq C \sum_{j \geq 0} C\left(\partial_{k} \varphi, j, N\right) b^{-N j} F_{\varphi}\left(\cdot, b^{j} t\right)_{N,\left(b^{j} t\right)^{-1}}^{* *}(x)+C D\left(\Xi_{k}, N\right) F_{\varphi}(\cdot, t)_{N, t^{-1}}^{* *}(x) .
\end{aligned}
$$

Taking the supremum in $z$ over $\mathbb{R}^{n}$, we reach the conclusion.

Let $\varphi \in B$. Then we have

$$
\sup _{j \geq 0} C_{\varphi}(\nabla \varphi, j, L) b^{-\tau j}<\infty
$$

for all $L, \tau>0$, where we write $\nabla \varphi=\left(\partial_{1} \varphi, \ldots, \partial_{n} \varphi\right), C_{\varphi}(\nabla \varphi, j, L)=\sum_{k=1}^{n} C_{\varphi}\left(\partial_{k} \varphi, j, L\right)$ and

$$
D_{\varphi}(L)<\infty
$$

for all $L>0$, where $D_{\varphi}(L)=\sum_{k=1}^{n} D_{\varphi}\left(\Xi_{k}, L\right)$. Let $\psi \in \mathcal{S}\left(\mathbb{R}^{n}\right)$. Then, we also note that

$$
\sup _{j: b j \leq r_{2}} C_{\varphi}(\psi, j, L) b^{-\tau j}<\infty \quad \text { for any } L, \tau>0 .
$$

These results are in Lemma 2.9 below, which will be used in what follows.

We consider a ball in $\mathbb{R}^{n}$ with center $x$ and radius $t$ relative to $\rho$ defined by

$$
B(x, t)=\left\{y \in \mathbb{R}^{n}: \rho(x-y)<t\right\} .
$$

We say that a weight function $w$ belongs to the class $A_{p}, 1<p<\infty$, of Muckenhoupt if

$$
[w]_{A_{p}}=\sup _{B}\left(|B|^{-1} \int_{B} w(x) d x\right)\left(|B|^{-1} \int_{B} w(x)^{-1 /(p-1)} d x\right)^{p-1}<\infty,
$$

where the supremum is taken over all balls $B$ in $\mathbb{R}^{n}$ and $|B|$ denotes the Lebesgue measure of $B$. Let $A_{\infty}=\cup_{p>1} A_{p}$. Also, we define the class $A_{1}$ to be the family of weight functions $w$ such that $M(w) \leq C w$ almost everywhere. We denote by $[w]_{A_{1}}$ the infimum of all such $C$. Here, $M$ is the Hardy-Littlewood maximal operator relative to $\rho$

$$
M(f)(x)=\sup _{x \in B}|B|^{-1} \int_{B}|f(y)| d y,
$$

where the supremum is taken over all balls $B$ in $\mathbb{R}^{n}$ containing $x$. (See 2, 10, )

For a weight $w$, we denote by $\|f\|_{p, w}$ the weighted $L^{p}$ norm

$$
\left(\int_{\mathbb{R}^{n}}|f(x)|^{p} w(x) d x\right)^{1 / p}
$$


Then we have the following result.

Theorem 2.4. Let $\varphi \in B$. Suppose that $0<p, q<\infty$ and $w \in A_{\infty}$. Let $\psi \in \mathcal{S}\left(\mathbb{R}^{n}\right)$. Suppose that $\hat{\psi}=0$ in a neighborhood of the origin. Then

$$
\left\|\left(\int_{0}^{\infty}\left|f * \psi_{t}\right|^{q} \frac{d t}{t}\right)^{1 / q}\right\|_{p, w} \leq C\left\|\left(\int_{0}^{\infty}\left|f * \varphi_{t}\right|^{q} \frac{d t}{t}\right)^{1 / q}\right\|_{p, w}
$$

for $f \in \mathcal{S}\left(\mathbb{R}^{n}\right)$ with a positive constant $C$ independent of $f$.

To prove Theorem 2.4 we first show the following.

Lemma 2.5. Let $0<q<\infty, N>0$. Suppose that $\varphi \in B, f \in \mathcal{S}\left(\mathbb{R}^{n}\right)$. Then

$$
\int_{0}^{\infty} F(\varphi, f)(\cdot, t)_{N, t^{-1}}^{* *}(x)^{q} \frac{d t}{t} \leq C \int_{0}^{\infty} M\left(\left|f * \varphi_{t}\right|^{r}\right)(x)^{q / r} \frac{d t}{t}, \quad r=\gamma / N .
$$

We apply the next result to show Lemma 2.5.

Lemma 2.6. Let $N=\gamma / r, r>0$ and let $\varphi \in L^{1}\left(\mathbb{R}^{n}\right), f \in \mathcal{S}\left(\mathbb{R}^{n}\right)$. Suppose that $\varphi$ satisfies (2.1). Then

$$
\left(f * \varphi_{t}\right)_{N, t^{-1}}^{* *}(x) \leq C \delta^{-N} M\left(\left|f * \varphi_{t}\right|^{r}\right)(x)^{1 / r}+C \delta\left|f *(\nabla \varphi)_{t}\right|_{N, t^{-1}}^{* *}(x)
$$

for all $\delta \in(0,1]$ with a constant $C$ independent of $\delta$ and $t>0$, where $f *(\nabla \varphi)_{t}=$ $\left(f *\left(\partial_{1} \varphi\right)_{t}, \ldots, f *\left(\partial_{n} \varphi\right)_{t}\right)$.

To prove Lemma 2.6. we use the following.

Lemma 2.7. Let $F \in C^{1}\left(\mathbb{R}^{n}\right)$ and $R>0, N=\gamma / r, r>0$. Then

$$
F_{N, 1}^{* *}(x) \leq C \delta^{-N} M\left(|F|^{r}\right)(x)^{1 / r}+C \delta|\nabla F|_{N, 1}^{* *}(x)
$$

for all $\delta \in(0,1]$ with a constant $C$ independent of $\delta$, where $F_{N, R}^{* *}$ is as in (2.10).

Proof. Let $f_{B(x, t)} f(y) d y=|B(x, t)|^{-1} \int_{B(x, t)} f(y) d y$. Then, for $\delta \in(0,1], r>0$ and $x, z \in \mathbb{R}^{n}$ we write

$$
|F(x-z)|=\left(f_{B(x-z, \delta)}|F(y)+(F(x-z)-F(y))|^{r} d y\right)^{1 / r} .
$$

This is bounded by

$$
C_{r}\left(f_{B(x-z, \delta)}|F(y)|^{r} d y\right)^{1 / r}+C_{r}\left(f_{B(x-z, \delta)}|F(x-z)-F(y)|^{r} d y\right)^{1 / r}
$$

where $C_{r}=1$ if $r \geq 1$ and $C_{r}=2^{-1+1 / r}$ if $0<r<1$. Thus we have

$$
|F(x-z)| \leq C_{r}\left(f_{B(x-z, \delta)}|F(y)|^{r} d y\right)^{1 / r}+C_{r} \sup _{y: \rho(x-z-y)<\delta}|x-z-y||\nabla F(y)| .
$$

If $|x-z-y| \leq 1,|x-z-y| \leq \rho(x-z-y)$ by (P.3). So, we see that

$$
|F(x-z)| \leq C_{r}\left(f_{B(x-z, \delta)}|F(y)|^{r} d y\right)^{1 / r}+C_{r} \sup _{y: \rho(x-z-y)<\delta} \delta|\nabla F(y)| .
$$


If $\rho(x-z-y)<\delta, \rho(x-y)<\delta+\rho(z)$. Therefore

$$
\begin{aligned}
|\nabla F(y)| & \leq \frac{|\nabla F(x+(y-x))|}{(1+\rho(x-y))^{N}}(1+\delta+\rho(z))^{N} \\
& \leq|\nabla F|_{N, 1}^{* *}(x)(1+\delta+\rho(z))^{N} \\
& \leq 2^{N}|\nabla F|_{N, 1}^{* *}(x)(1+\rho(z))^{N} .
\end{aligned}
$$

Thus

$$
\sup _{y: \rho(x-z-y)<\delta} \delta|\nabla F(y)| \leq 2^{N} \delta|\nabla F|_{N, 1}^{* *}(x)(1+\rho(z))^{N} .
$$

On the other hand,

$$
\begin{aligned}
& \left(f_{B(x-z, \delta)}|F(y)|^{r} d y\right)^{1 / r} \leq\left(\delta^{-\gamma}(\delta+\rho(z))^{\gamma} f_{B(x, \delta+\rho(z))}|F(y)|^{r} d y\right)^{1 / r} \\
& \leq \delta^{-\gamma / r}(\delta+\rho(z))^{\gamma / r} M\left(|F|^{r}\right)(x)^{1 / r} \\
& \leq \delta^{-\gamma / r}(1+\rho(z))^{\gamma / r} M\left(|F|^{r}\right)(x)^{1 / r} .
\end{aligned}
$$

By (2.14), (2.15) and (2.16), we have

$$
|F(x-z)| \leq C_{r} \delta^{-\gamma / r}(1+\rho(z))^{\gamma / r} M\left(|F|^{r}\right)(x)^{1 / r}+2^{N} C_{r} \delta|\nabla F|_{N, 1}^{* *}(x)(1+\rho(z))^{N} \text {. }
$$

Thus, if $N=\gamma / r$, we see that

$$
\frac{|F(x-z)|}{(1+\rho(z))^{N}} \leq C_{r} \delta^{-N} M\left(|F|^{r}\right)(x)^{1 / r}+2^{N} C_{r} \delta|\nabla F|_{N, 1}^{* *}(x) .
$$

Taking the supremum in $z$ over $\mathbb{R}^{n}$, we get the desired estimate.

Proof of Lemma 2.6, Let $\left(T_{t} f\right)(x)=f\left(A_{t} x\right)$. Then we note the following.

(T.1) $\left(T_{t} F_{N, R}^{* *}\right)(x)=\left(T_{t} F\right)_{N, t R}^{* *}(x)$.

(T.2) $T_{t}(f * g)(x)=t^{\gamma}\left(T_{t} f\right) *\left(T_{t} g\right)(x)$.

(T.3) $T_{t}(M(f))(x)=M\left(T_{t} f\right)(x)$.

By (T.1) and (T.2) we have

$$
T_{t}\left(\left(f * \varphi_{t}\right)_{N, t^{-1}}^{* *}\right)(x)=\left(T_{t} f * \varphi\right)_{N, 1}^{* *}(x) .
$$

Using Lemma 2.7 we see that

$$
\left(T_{t} f * \varphi\right)_{N, 1}^{* *}(x) \leq C \delta^{-N} M\left(\left|T_{t} f * \varphi\right|^{r}\right)(x)^{1 / r}+C \delta\left|T_{t} f * \nabla \varphi\right|_{N, 1}^{* *}(x) .
$$

Applying $T_{t^{-1}}$ to (2.17), we have

$$
\left(f * \varphi_{t}\right)_{N, t^{-1}}^{* *}(x) \leq C \delta^{-N} T_{t^{-1}}\left(M\left(\left|T_{t} f * \varphi\right|^{r}\right)(x)^{1 / r}\right)+C \delta T_{t^{-1}}\left(\left|T_{t} f * \nabla \varphi\right|_{N, 1}^{* *}\right)(x) .
$$

From (T.2) and (T.3), it follows that

$$
T_{t^{-1}}\left(M\left(\left|T_{t} f * \varphi\right|^{r}\right)(x)^{1 / r}\right)=M\left(\left|f * \varphi_{t}\right|^{r}\right)(x)^{1 / r} .
$$

Also, (T.1) and (T.2) imply

$$
T_{t^{-1}}\left(\left|T_{t} f * \nabla \varphi\right|_{N, 1}^{* *}\right)(x)=\left|f *(\nabla \varphi)_{t}\right|_{N, t^{-1}}^{* *}(x) .
$$

Using (2.19) and (2.20) in (2.18), we get the conclusion of Lemma 2.6. 
Proof of Lemma 2.5. Lemma 2.6 implies that

$$
F(\varphi, f)(\cdot, t)_{N, t^{-1}}^{* *}(x) \leq C \delta^{-N} M\left(\left|f * \varphi_{t}\right|^{r}\right)(x)^{1 / r}+C \delta\left|f *(\nabla \varphi)_{t}\right|_{N, t^{-1}}^{* *}(x),
$$

where $r=\gamma / N$. Applying Lemma 2.3, we have

$$
\begin{aligned}
& \left|f *(\nabla \varphi)_{t}\right|_{N, t^{-1}}^{* *}(x) \\
\leq & C \sum_{j \geq 0} C_{\varphi}(\nabla \varphi, j, N) b^{-j N} F(\varphi, f)\left(\cdot, b^{j} t\right)_{N,\left(b^{j} t\right)^{-1}}^{* *}(x)+C D_{\varphi}(N) F(\varphi, f)(\cdot, t)_{N, t^{-1}}^{* *}(x) .
\end{aligned}
$$

Thus by (2.21) and Hölder's inequality when $q>1$ we have

$$
\begin{aligned}
& F(\varphi, f)(\cdot, t)_{N, t^{-1}}^{* *}(x)^{q} \leq C \delta^{-N q} M\left(\left|f * \varphi_{t}\right|^{r}\right)(x)^{q / r} \\
& +C \delta^{q} \sum_{j \geq 0} C_{\varphi}(\nabla \varphi, j, N)^{q} b^{-j N q} b^{-\tau c_{q j} j} F(\varphi, f)\left(\cdot, b^{j} t\right)_{N,\left(b^{j} t\right)^{-1}}^{* *}(x)^{q} \\
& \quad+C \delta^{q} D_{\varphi}(N)^{q} F(\varphi, f)(\cdot, t)_{N, t^{-1}}^{* *}(x)^{q} .
\end{aligned}
$$

where $\tau>0, c_{q}=1$ when $q>1$ and $c_{q}=0$ when $0<q \leq 1$. Integrating both sides of the inequality (2.22) over $(0, \infty)$ with respect to the measure $d t / t$ and applying termwise integration on the right hand side, we see that

$$
\begin{aligned}
& \text { (2.23) } \int_{0}^{\infty} F(\varphi, f)(\cdot, t)_{N, t^{-1}}^{* *}(x)^{q} \frac{d t}{t} \leq C \delta^{-N q} \int_{0}^{\infty} M\left(\left|f * \varphi_{t}\right|^{r}\right)(x)^{q / r}(x) \frac{d t}{t} \\
& +C \delta^{q}\left[\sum_{j \geq 0} C_{\varphi}(\nabla \varphi, j, N)^{q} b^{-j N q} b^{-\tau c_{q} j}+D_{\varphi}(N)^{q}\right] \int_{0}^{\infty} F(\varphi, f)(\cdot, t)_{N, t^{-1}}^{* *}(x)^{q} \frac{d t}{t} .
\end{aligned}
$$

By (2.11) the sum in $j$ on the right hand side of (2.23) is finite. By (2.2) and (2.3) with $\alpha=0$ we easily see that the last integral on the right hand side of (2.23) is finite with $f \in \mathcal{S}\left(\mathbb{R}^{n}\right)$. Thus, along with (2.12) we see that the second term on the right hand side of (2.23) is finite. So, choosing $\delta$ sufficiently small, we get the conclusion.

We need the following version of the vector valued inequality for the HardyLittlewood maximal operator of Fefferman-Stein [7] with non-isotropic dilations and weights. The proof is essentially similar to the one in [7]. The case $P=E$ is stated in [16] (see [14] for related results).

Lemma 2.8. Let $1<\mu, \nu<\infty$ and $w \in A_{\nu}$. Then we have

$$
\left\|\left(\int_{0}^{\infty} M\left(F^{t}\right)(x)^{\mu} \frac{d t}{t}\right)^{1 / \mu}\right\|_{\nu, w} \leq C\left(\int_{\mathbb{R}^{n}}\left(\int_{0}^{\infty}|F(x, t)|^{\mu} \frac{d t}{t}\right)^{\nu / \mu} w(x) d x\right)^{1 / \nu}
$$

for appropriate functions $F(x, t)$ on $\mathbb{R}^{n} \times(0, \infty)$ with $F^{t}(x)=F(x, t)$.

Proof of Theorem 2.4. By a change of variables we may assume that $\hat{\psi}=0$ on $\{|\xi| \leq 1\}=\left\{\rho^{*}(\xi) \leq 1\right\}$. Define

$$
\hat{\zeta}(\xi)=1-\sum_{j: b j \leq r_{2}} \hat{\varphi}\left(A_{b^{j}}^{*} \xi\right) \hat{\eta}\left(A_{b^{j}}^{*} \xi\right)
$$


Then $\operatorname{supp}(\hat{\zeta}) \subset\left\{\rho^{*}(\xi) \leq 1\right\}, \hat{\zeta}=1$ in $\left\{\rho^{*}(\xi)<r_{1} / r_{2}\right\}$. Since $\hat{\psi}=0$ on $\{|\xi| \leq 1\}$, we have

$$
\begin{aligned}
\hat{\psi}(\xi) & =\sum_{j: b^{j} \leq r_{2}} \hat{\psi}(\xi) \hat{\varphi}\left(A_{b^{j}}^{*} \xi\right) \hat{\eta}\left(A_{b^{j}}^{*} \xi\right) \\
& =\sum_{j: b^{j} \leq r_{2}} \hat{\varphi}\left(A_{b^{j}}^{*} \xi\right) \mathcal{F}\left(\alpha^{\left(b^{j}\right)}\right)\left(A_{b^{j}}^{*} \xi\right),
\end{aligned}
$$

where $\alpha^{\left(b^{j}\right)}(x)=(\psi)_{b^{-j}} * \eta(x)$.

Thus by an easier version of arguments for the proof of Lemma 2.3 we see that

$$
|F(\psi, f)(x, t)| \leq C \sum_{j: b^{j} \leq r_{2}} C(\psi, j, N) F(\varphi, f)\left(\cdot, b^{j} t\right)_{N,\left(b^{j} t\right)^{-1}}^{* *}(x),
$$

from which it follows that

$$
|F(\psi, f)(x, t)|^{q} \leq C \sum_{j: b^{j} \leq r_{2}} C(\psi, j, N)^{q} b^{-\tau c_{q} j} F(\varphi, f)\left(\cdot, b^{j} t\right)_{N,\left(b^{j} t\right)^{-1}}^{*}(x)^{q},
$$

where $\tau>0$ and $c_{q}$ is as in (2.22). Integrating with the measure $d t / t$ over $(0, \infty)$, we have

$$
\begin{aligned}
& \int_{0}^{\infty}|F(\psi, f)(x, t)|^{q} \frac{d t}{t} \\
& \quad \leq C\left[\sum_{j: b^{j} \leq r_{2}} C(\psi, j, N)^{q} b^{-\tau c_{q} j}\right] \int_{0}^{\infty} F(\varphi, f)(\cdot, t)_{N, t^{-1}}^{* *}(x)^{q} \frac{d t}{t} .
\end{aligned}
$$

By (2.13) the series on the right hand side of (2.24) converges. Let $0<p, q<\infty$ and $w \in A_{\infty}$. If $N$ is sufficiently large so that $r=\gamma / N<q, p$ and $w \in A_{p N / \gamma}$, from (2.24) and Lemma 2.5) it follows that

$$
\begin{aligned}
\left\|\left(\int_{0}^{\infty}\left|f * \psi_{t}\right|^{q} \frac{d t}{t}\right)^{1 / q}\right\|_{p, w} & \leq C\left\|\left(\int_{0}^{\infty} M\left(\left|f * \varphi_{t}\right|^{r}\right)(x)^{q / r} \frac{d t}{t}\right)^{1 / q}\right\|_{p, w} \\
& =C\left\|\left(\int_{0}^{\infty} M\left(\left|f * \varphi_{t}\right|^{r}\right)(x)^{q / r} \frac{d t}{t}\right)^{r / q}\right\|_{p / r, w}^{1 / r} \\
& \leq C\left\|\left(\int_{0}^{\infty}\left|f * \varphi_{t}(x)\right|^{q} \frac{d t}{t}\right)^{1 / q}\right\|_{p, w},
\end{aligned}
$$

where the last inequality follows form Lemma 2.8. This completes the proof of Theorem 2.4.

To conclude this section, we give a proof of the following results used above.

Lemma 2.9. Let $\varphi \in B$. Suppose that $\psi \in L^{1}, \hat{\psi} \in C^{\infty}\left(\mathbb{R}^{n} \backslash\{0\}\right)$ and we have the estimates (2.3) with $\hat{\psi}$ in place of $\hat{\varphi}$ for all multi-indices $\alpha$ and all $\tau>0$. Let $L, J \geq 0$. Then

(1) $\sup _{j: b^{j} \leq J} C_{\varphi}(\psi, j, L) b^{-j \tau}<\infty$ for any $\tau>0$, where $C_{\varphi}(\psi, j, L)=C(\psi, j, L)$ is as in (2.8) with $\psi$ in place of $\partial_{k} \varphi$.

(2) $D_{\varphi}\left(\Xi_{k}, L\right)<\infty, 1 \leq k \leq n$, where $\Xi_{k}(\xi)=2 \pi i \xi_{k}$ as above and $D_{\varphi}\left(\Xi_{k}, L\right)=$ $D\left(\Xi_{k}, L\right)$ is as in (2.9). 
Proof. To prove part (1), since $1+\rho(x) \leq c(1+|x|)$ by (P.4), we have

$$
\begin{aligned}
& (1+|x|)^{[n / 2]+1} C_{0}(\psi, t, L, x) \\
\leq & C\left|\int \hat{\psi}\left(A_{t^{-1}}^{*} \xi\right) \hat{\eta}(\xi) e^{2 \pi i\langle x, \xi\rangle} d \xi\right|+C \sup _{|\alpha|=L+[n / 2]+1}\left|\int \partial_{\xi}^{\alpha}\left[\hat{\psi}\left(A_{t^{-1}}^{*} \xi\right) \hat{\eta}(\xi)\right] e^{2 \pi i\langle x, \xi\rangle} d \xi\right|,
\end{aligned}
$$

where $C_{0}(\psi, t, L, x)$ is as in (2.6) with $\psi$ in place of $\partial_{k} \varphi$ and $[a]$ denotes the largest integer not exceeding $a$. We recall that $\hat{\eta} \in C^{\infty}\left(\mathbb{R}^{n}\right)$ with support in $\left\{r_{1}<\rho^{*}(\xi)<\right.$ $\left.r_{2}\right\}$, which is in Lemma 2.1. It is known that $\left\|A_{t^{-1}}^{*}\right\| \leq t^{-\kappa}$ for $t \in(0,1]$ with some $\kappa \geq 1$. Thus (2.3) for $\hat{\psi}$ implies

$$
\left|\partial_{\xi}^{\alpha}\left[\hat{\psi}\left(A_{t^{-1}}^{*} \xi\right) \hat{\eta}(\xi)\right]\right| \leq C_{\alpha, M} t^{\tau}, \quad 0<t \leq M
$$

for any $M>0$, if $|\alpha|=L+[n / 2]+1$ or $\alpha=0$. Thus

$$
C_{0}(\psi, t, L, x) \leq C(1+|x|)^{-[n / 2]-1} G(x)
$$

with some $G \in L^{2}$ such that $\|G\|_{2} \leq C t^{\tau}$, and hence, the Schwarz inequality implies

$$
\int_{\mathbb{R}^{n}} C_{0}(\psi, t, L, x) d x \leq C t^{\tau},
$$

since $[n / 2]+1>n / 2$. From (2.26) with $t=b^{j}$ we obtain the conclusion of part (1).

Similarly, we can see that

$$
\int_{\mathbb{R}^{n}} D\left(\Xi_{k}, L, x\right) d x<\infty
$$

where $D\left(\Xi_{k}, L, x\right)$ is as in (2.7). This completes the proof of part (2).

\section{Littlewood-Paley functions and Parabolic Hardy SPaCes}

As an application of Theorem 2.4 we have the following.

Corollary 3.1. Let $0<p \leq 1$. Suppose that $\varphi \in B$. Then we have

$$
\|f\|_{H^{p}} \leq C_{p}\left\|g_{\varphi}(f)\right\|_{p}
$$

for $f \in H^{p}\left(\mathbb{R}^{n}\right) \cap \mathcal{S}\left(\mathbb{R}^{n}\right)$ with a positive constant $C_{p}$ independent of $f$.

Let $\mathcal{H}$ be the Hilbert space of functions $u(t)$ on $\mathbb{R}_{+}$such that $\|u\|_{\mathcal{H}}=\left(\int_{0}^{\infty}|u(t)|^{2} d t / t\right)^{1 / 2}<$ $\infty$. Let $L_{\mathcal{H}}^{q}\left(\mathbb{R}^{n}\right)$ be the Lebesgue space of functions $h(y, t)$ with the norm

$$
\|h\|_{q, \mathcal{H}}=\left(\int_{\mathbb{R}^{n}}\left\|h^{y}\right\|_{\mathcal{H}}^{q} d y\right)^{1 / q}
$$

where $h^{y}(t)=h(y, t)$.

Let $0<p \leq 1$. We consider the parabolic Hardy space of functions on $\mathbb{R}^{n}$ with values in $\mathcal{H}$, which is denoted by $H_{\mathcal{H}}^{p}\left(\mathbb{R}^{n}\right)$. Choose $\Phi \in \mathcal{S}\left(\mathbb{R}^{n}\right)$ as in the definition of $H^{p}$ in Section 1. Let $h \in L_{\mathcal{H}}^{2}\left(\mathbb{R}^{n}\right)$. We say $h \in H_{\mathcal{H}}^{p}\left(\mathbb{R}^{n}\right)$ if $\|h\|_{H_{\mathcal{H}}^{p}}=\left\|h^{*}\right\|_{L^{p}}<\infty$ with

$$
h^{*}(x)=\sup _{s>0}\left(\int_{0}^{\infty}\left|\Phi_{s} * h^{t}(x)\right|^{2} \frac{d t}{t}\right)^{1 / 2},
$$

where we write $h^{t}(x)=h(x, t)$.

To prove Corollary 3.1 we need the following. 
Lemma 3.2. Let $\hat{\psi}$ be a function of $\mathcal{S}\left(\mathbb{R}^{n}\right)$ with support in $\left\{1 \leq \rho^{*}(\xi) \leq 2\right\}$. Suppose that

$$
\int_{0}^{\infty}\left|\hat{\psi}\left(A_{t}^{*} \xi\right)\right|^{2} \frac{d t}{t}=1 \quad \text { for all } \xi \neq 0 .
$$

Let $F(y, t)=f * \psi_{t}(y)$ with $f \in H^{p}\left(\mathbb{R}^{n}\right) \cap \mathcal{S}\left(\mathbb{R}^{n}\right), 0<p \leq 1$. Then $F \in H_{\mathcal{H}}^{p}\left(\mathbb{R}^{n}\right)$ and

$$
\|f\|_{H^{p}} \leq C\|F\|_{H_{\mathcal{H}}^{p}} .
$$

Define

$$
E_{\psi}^{\epsilon}(h)(x)=\int_{0}^{\infty} \int_{\mathbb{R}^{n}} \psi_{t}(x-y) h_{(\epsilon)}(y, t) d y \frac{d t}{t},
$$

where $h \in L_{\mathcal{H}}^{2}$ and $h_{(\epsilon)}(y, t)=h(y, t) \chi_{\left(\epsilon, \epsilon^{-1}\right)}(t), 0<\epsilon<1$, and we assume that $\psi \in L^{1}\left(\mathbb{R}^{n}\right)$ with the cancellation (1.2). Here $\chi_{S}$ denotes the characteristic function of a set $S$.

We apply the following result in proving Lemma 3.2 .

Lemma 3.3. Suppose that $\psi \in \mathcal{S}\left(\mathbb{R}^{n}\right)$ and $\operatorname{supp} \hat{\psi} \subset\left\{1 \leq \rho^{*}(\xi) \leq 2\right\}$. Then

$$
\sup _{\epsilon \in(0,1)}\left\|E_{\psi}^{\epsilon}(h)\right\|_{H^{p}} \leq C\|h\|_{H_{\mathcal{H}}^{p}}, \quad 0<p \leq 1 .
$$

Let $a$ be a $(p, \infty)$ atom in $H_{\mathcal{H}}^{p}\left(\mathbb{R}^{n}\right)$ :

(i) $\left(\int_{0}^{\infty}|a(x, t)|^{2} d t / t\right)^{1 / 2} \leq|B|^{-1 / p}$, where $B$ is a ball in $\mathbb{R}^{n}$ with respect to $\rho$;

(ii) $\operatorname{supp}(a(\cdot, t)) \subset B$ for all $t>0$, where $B$ is as in $(i)$;

(iii) $\int_{\mathbb{R}^{n}} a(x, t) x^{\alpha} d x=0$ for all $t>0$ and $\alpha$ such that $|\alpha| \leq[\gamma(1 / p-1)]$, where $\alpha=\left(\alpha_{1}, \ldots, \alpha_{n}\right)$ is a multi-index with $x^{\alpha}=x_{1}^{\alpha_{1}} \ldots x_{n}^{\alpha_{n}}$.

To prove Lemma 3.3 we use the following atomic decomposition.

Lemma 3.4. Let $h \in L_{\mathcal{H}}^{2}\left(\mathbb{R}^{n}\right)$. Suppose that $h \in H_{\mathcal{H}}^{p}\left(\mathbb{R}^{n}\right)$. Then we can find a sequence $\left\{a_{k}\right\}$ of $(p, \infty)$ atoms in $H_{\mathcal{H}}^{p}\left(\mathbb{R}^{n}\right)$ and a sequence $\left\{\lambda_{k}\right\}$ of positive numbers such that $h=\sum_{k=1}^{\infty} \lambda_{k} a_{k}$ in $H_{\mathcal{H}}^{p}\left(\mathbb{R}^{n}\right)$ and in $L_{\mathcal{H}}^{2}\left(\mathbb{R}^{n}\right)$ and such that $\sum_{k=1}^{\infty} \lambda_{k}^{p} \leq$ $C\|h\|_{H_{\mathcal{H}}^{p}}^{p}$ with a constant $C$ independent of $h$.

We can find in [12] a proof of the atomic decomposition for $H^{p}\left(\mathbb{R}^{n}\right)$ (see also [3], [10] and [18). The vector valued case can be treated similarly.

Proof of Lemma 3.3. Let $a$ be a $(p, \infty)$ atom in $H_{\mathcal{H}}^{p}\left(\mathbb{R}^{n}\right)$ supported on the ball $B$ of the definition of the atom. We choose a non-negative $C^{\infty}$ function $\Phi$ on $\mathbb{R}^{n}$ supported on $\{|x|<1\}$ with $\int \Phi(x) d x=1$. We prove

$$
\int_{\mathbb{R}^{n}} \sup _{s>0}\left|\Phi_{s} * E_{\psi}^{\epsilon}(a)(x)\right|^{p} d x \leq C .
$$

To show this, by applying translation and dilation arguments, we may assume that $B=B(0,1)$. Let $\widetilde{B}=B(0,2)$. Then $2 \rho(y) \leq \rho(x)$ if $y \in B$ and $x \in \mathbb{R}^{n} \backslash \widetilde{B}$. Let $\Psi_{s, t}=\Phi_{s} * \psi_{t}, s, t>0$. We note that $\Phi_{s} * \psi_{t}=\left(\Phi_{s / t} * \psi\right)_{t}$ and $\Phi_{u} * \psi, u>0$, belongs to a bounded subset of the topological vector space $\mathcal{S}\left(\mathbb{R}^{n}\right)$, which can easily be seen by noting that $\mathcal{F}\left(\Phi_{u} * \psi\right)(\xi)=\hat{\Phi}\left(A_{u}^{*} \xi\right) \hat{\psi}(\xi)$ and recalling that $\hat{\psi}(\xi)$ is supported on $\left\{1 \leq \rho^{*}(\xi) \leq 2\right\}$.

Let $P_{x}(y)$ be the Taylor polynomial in $y$ of order $M=[\gamma(1 / p-1)]$ at 0 for $\Phi_{s / t} * \psi(x-y)$. Then, if $\rho(x) \geq 2 \rho(y)$ and we have

$$
\left|\Phi_{s / t} * \psi(x-y)-P_{x}(y)\right| \leq C|y|^{M+1}(1+\rho(x))^{-L},
$$


where $L$ is sufficiently large, which will be specified below, and the constant $C$ is independent of $s, t, x, y$. This implies

$$
\left|\Psi_{s, t}(x-y)-t^{-\gamma} P_{A_{t^{-1}} x}\left(A_{t^{-1}} y\right)\right| \leq C t^{-\gamma}\left|A_{t^{-1}} y\right|^{M+1}(1+\rho(x) / t)^{-L} .
$$

Thus, using the properties of an atom and the Schwarz inequality, for $x \in \mathbb{R}^{n} \backslash \widetilde{B}$ we see that

$$
\begin{aligned}
& \left|\Phi_{s} * E_{\psi}^{\epsilon}(a)(x)\right|=\left|\iint\left(\Psi_{s, t}(x-y)-t^{-\gamma} P_{A_{t^{-1}} x}\left(A_{t^{-1}} y\right)\right) a_{(\epsilon)}(y, t) d y \frac{d t}{t}\right| \\
& \leq \int_{B}\left(\int_{0}^{\infty}\left|\Psi_{s, t}(x-y)-t^{-\gamma} P_{A_{t^{-1}} x}\left(A_{t^{-1}} y\right)\right|^{2} \frac{d t}{t}\right)^{1 / 2}\left(\int_{0}^{\infty}|a(y, t)|^{2} \frac{d t}{t}\right)^{1 / 2} d y \\
& \leq C|B|^{-1 / p} \int_{B}\left(\int_{0}^{\infty}\left(t^{-\gamma}\left|A_{t^{-1}} y\right|^{M+1}(1+\rho(x) / t)^{-L}\right)^{2} \frac{d t}{t}\right)^{1 / 2} d y .
\end{aligned}
$$

Now we show that

$$
I(x, y):=\int_{0}^{\infty}\left(t^{-\gamma}\left|A_{t^{-1}} y\right|^{M+1}(1+\rho(x) / t)^{-L}\right)^{2} \frac{d t}{t} \leq C \rho(x)^{-2(\gamma+M+1)}
$$

for $y \in B, x \in \mathbb{R}^{n} \backslash \widetilde{B}$, if $L$ is sufficiently large. We first see that

$$
I(x, y)=\rho(x)^{-2 \gamma} \int_{0}^{\infty}\left(t^{-\gamma}\left|A_{(\rho(x) t)^{-1}} y\right|^{M+1}\left(1+t^{-1}\right)^{-L}\right)^{2} \frac{d t}{t} .
$$

By (P.6) we have

$$
\begin{aligned}
& \int_{\rho(x)^{-1}}^{\infty}\left(t^{-\gamma}\left|A_{(\rho(x) t)^{-1}} y\right|^{M+1}\left(1+t^{-1}\right)^{-L}\right)^{2} \frac{d t}{t} \\
& \leq C|y|^{2(M+1)} \rho(x)^{-2(M+1)} \int_{\rho(x)^{-1}}^{\infty} t^{-2(\gamma+M+1)}\left(1+t^{-1}\right)^{-2 L} \frac{d t}{t} \\
& \leq C|y|^{2(M+1)} \rho(x)^{-2(M+1)}
\end{aligned}
$$

if $L>\gamma+M+1$. If $s \geq 1,\left|A_{s} y\right| \leq C s^{\kappa}|y|$ for some $\kappa \geq 1$. Thus

$$
\begin{aligned}
& \int_{0}^{\rho(x)^{-1}}\left(t^{-\gamma}\left|A_{(\rho(x) t)^{-1}} y\right|^{M+1}\left(1+t^{-1}\right)^{-L}\right)^{2} \frac{d t}{t} \\
& \leq C|y|^{2(M+1)} \rho(x)^{-2 \kappa(M+1)} \int_{0}^{\rho(x)^{-1}} t^{-2(\gamma+\kappa(M+1))}\left(1+t^{-1}\right)^{-2 L} \frac{d t}{t} \\
& \leq C|y|^{2(M+1)} \rho(x)^{-2 \kappa(M+1)},
\end{aligned}
$$

if $L>\gamma+\kappa(M+1)$. By (3.4), (3.5) and (3.6) we obtain (3.3). From (3.2) and (3.3) we have

$$
\left|\Phi_{s} * E_{\psi}^{\epsilon}(a)(x)\right| \leq C \rho(x)^{-(\gamma+M+1)}
$$

for $x \in \mathbb{R}^{n} \backslash \widetilde{B}$.

Since $p>\gamma /(\gamma+M+1)$, it follows that

$$
\int_{\mathbb{R}^{n} \backslash \widetilde{B}} \sup _{s>0}\left|\Phi_{s} * E_{\psi}^{\epsilon}(a)(x)\right|^{p} d x \leq C \int_{\mathbb{R}^{n} \backslash \widetilde{B}} \rho(x)^{-p(\gamma+M+1)} d x \leq C
$$

(see [6]). 
Using $\int_{0}^{\infty}\left|\hat{\psi}\left(A_{t}^{*} \xi\right)\right|^{2} d t / t \leq C$, by duality we can easily see that

$$
\sup _{\epsilon \in(0,1)}\left\|E_{\psi}^{\epsilon}(h)\right\|_{2} \leq C\|h\|_{L_{\mathcal{H}}^{2}}, \quad h \in L_{\mathcal{H}}^{2}\left(\mathbb{R}^{n}\right) .
$$

So, by Hölder's inequality and the properties (i), (ii) of $a$, we get

$$
\begin{aligned}
\int_{\widetilde{B}} \sup _{s>0}\left|\Phi_{s} * E_{\psi}^{\epsilon}(a)(x)\right|^{p} d x & \leq C\left(\int_{\widetilde{B}}\left|M\left(E_{\psi}^{\epsilon}(a)\right)(x)\right|^{2} d x\right)^{p / 2} \\
& \leq C\left(\int_{B} \int_{0}^{\infty}|a(y, t)|^{2} \frac{d t}{t} d y\right)^{p / 2} \\
& \leq C .
\end{aligned}
$$

Combining (3.7) and (3.8), we have (3.1). By Lemma 3.4 and (3.1) we can prove

$$
\int_{\mathbb{R}^{n}} \sup _{s>0}\left|\Phi_{s} * E_{\psi}^{\epsilon}(h)(x)\right|^{p} d x \leq C\|h\|_{H_{\mathcal{H}}^{p}}^{p} .
$$

This completes the proof.

Proof of Lemma 3.2. By using the atomic decomposition for $H^{p}\left(\mathbb{R}^{n}\right)$, we can prove the fact that $F \in H_{\mathcal{H}}^{p}\left(\mathbb{R}^{n}\right)$ similarly to the proof of Lemma 3.3 (see [19, Lemma $3.6]$ ).

We note that

$$
E_{\widetilde{\psi}}^{\epsilon}(F)(x)=\int_{\epsilon}^{\epsilon^{-1}} \int_{\mathbb{R}^{n}} \psi_{t} * f(y) \bar{\psi}_{t}(y-x) d y \frac{d t}{t}=\int_{\mathbb{R}^{n}} \Psi^{(\epsilon)}(x-z) f(z) d z,
$$

where

$$
\Psi^{(\epsilon)}(x)=\int_{\epsilon}^{\epsilon^{-1}} \int_{\mathbb{R}^{n}} \psi_{t}(x+y) \bar{\psi}_{t}(y) d y \frac{d t}{t}
$$

We have

$$
\widehat{\Psi^{(\epsilon)}}(\xi)=\int_{\epsilon}^{\epsilon^{-1}} \hat{\psi}\left(A_{t}^{*} \xi\right) \widehat{\bar{\psi}}\left(-A_{t}^{*} \xi\right) \frac{d t}{t}=\int_{\epsilon}^{\epsilon^{-1}}\left|\hat{\psi}\left(A_{t}^{*} \xi\right)\right|^{2} \frac{d t}{t} .
$$

This and Lemma 3.3 imply

$$
\|f\|_{H^{p}} \leq C \liminf _{\epsilon \rightarrow 0}\left\|E_{\widetilde{\psi}}^{\epsilon}(F)\right\|_{H^{p}} \leq C\|F\|_{H_{\mathcal{H}}^{p}} .
$$

We also need the following result to prove Corollary 3.1 .

Lemma 3.5. Let $\eta \in \mathcal{S}\left(\mathbb{R}^{n}\right)$ satisfy $\operatorname{supp}(\hat{\eta}) \subset\left\{1 / 2 \leq \rho^{*}(\xi) \leq 4\right\}$ and $\hat{\eta}(\xi)=1$ on $\left\{1 \leq \rho^{*}(\xi) \leq 2\right\}$. Let $\psi$ be as in Lemma 3.2. Suppose that $\Phi \in \mathcal{S}\left(\mathbb{R}^{n}\right)$ satisfies $\int_{\mathbb{R}^{n}} \Phi(x) d x=1$ and $\operatorname{supp}(\Phi) \subset B(0,1)$. Then for $p, q>0$ and $f \in \mathcal{S}\left(\mathbb{R}^{n}\right)$ we have

$$
\left\|\left(\int_{0}^{\infty} \sup _{s>0}\left|\Phi_{s} * \psi_{t} * f\right|^{q} \frac{d t}{t}\right)^{1 / q}\right\|_{p} \leq C\left\|\left(\int_{0}^{\infty}\left|\eta_{t} * f\right|^{q} \frac{d t}{t}\right)^{1 / q}\right\|_{p} .
$$


Proof. Since $\hat{\Phi}\left(A_{s}^{*} \xi\right) \hat{\psi}\left(A_{t}^{*} \xi\right)=\hat{\Phi}\left(A_{s}^{*} \xi\right) \hat{\psi}\left(A_{t}^{*} \xi\right) \hat{\eta}\left(A_{t}^{*} \xi\right)$, we have

$$
\begin{aligned}
\left|\Phi_{s} * \psi_{t} * f(x)\right| & \leq\left(f * \eta_{t}\right)_{N, t^{-1}}^{* *}(x) \int_{\mathbb{R}^{n}}\left|\Phi_{s} * \psi_{t}(w)\right|\left(1+t^{-1} \rho(w)\right)^{N} d w \\
& =\left(f * \eta_{t}\right)_{N, t^{-1}}^{* *}(x) \int_{\mathbb{R}^{n}}\left|\Phi_{s / t} * \psi(w)\right|(1+\rho(w))^{N} d w \\
& \leq C_{N}\left(f * \eta_{t}\right)_{N, t^{-1}}^{* *}(x)
\end{aligned}
$$

for any $N>0$, where $C_{N}$ is independent of $s, t$. This follows from the observation that $\Phi_{s / t} * \psi, s, t>0$, belongs to a bounded subset of the topological vector space $\mathcal{S}\left(\mathbb{R}^{n}\right)$, as in the proof of Lemma 3.3. Thus

$$
\left(\int_{0}^{\infty} \sup _{s>0}\left|\Phi_{s} * \psi_{t} * f(x)\right|^{q} \frac{d t}{t}\right)^{1 / q} \leq C\left(\int_{0}^{\infty}\left|\left(f * \eta_{t}\right)_{N, t^{-1}}^{* *}(x)\right|^{q} \frac{d t}{t}\right)^{1 / q} .
$$

By (3.9) and Lemma 2.5 with $\eta$ in place of $\varphi$, we have

$$
\left(\int_{0}^{\infty} \sup _{s>0}\left|\Phi_{s} * \psi_{t} * f(x)\right|^{q} \frac{d t}{t}\right)^{1 / q} \leq C\left(\int_{0}^{\infty} M\left(\left|f * \eta_{t}\right|^{r}\right)(x)(x)^{q / r} \frac{d t}{t}\right)^{1 / q},
$$

where $N=\gamma / r$. This and Lemma 2.8 prove Lemma 3.5 as in (2.25).

Proof of Corollary 3.1. Let $\eta$ be as in Lemma3.5. Applying Lemma 3.2 and Lemma 3.5 with $q=2$ and $p \in(0,1]$, we see that

$$
\|f\|_{H^{p}} \leq C\left\|g_{\eta}(f)\right\|_{p}, \quad f \in H^{p}\left(\mathbb{R}^{n}\right) \cap \mathcal{S}\left(\mathbb{R}^{n}\right),
$$

which combined with Theorem 2.4 with $q=2, p \in(0,1], w=1$ and with $\eta$ in place of $\psi$ proves Corollary 3.1

Proof of Theorem 1.1. Let $\varphi$ be as in Theorem 1.1. Let $0<p \leq 1$. The inequality

$$
\left\|g_{\varphi}(f)\right\|_{p} \leq C\|f\|_{H^{p}}
$$

can be proved similarly to the proof of the statement $F \in H_{\mathcal{H}}^{p}\left(\mathbb{R}^{n}\right)$ in Lemma 3.2 for $f \in H^{p}\left(\mathbb{R}^{n}\right) \cap \mathcal{S}\left(\mathbb{R}^{n}\right)$ by using the atomic decomposition for $H^{p}\left(\mathbb{R}^{n}\right)$. This and Corollary 3.1 imply

$$
c_{1}\|f\|_{H^{p}} \leq\left\|g_{\varphi}(f)\right\|_{p} \leq c_{2}\|f\|_{H^{p}}
$$

for $f \in H^{p}\left(\mathbb{R}^{n}\right) \cap \mathcal{S}\left(\mathbb{R}^{n}\right)$, from which the conclusion of Theorem 1.1 follows by arguments similar to the one in [19, pp. 149-150], since $H^{p}\left(\mathbb{R}^{n}\right) \cap \mathcal{S}\left(\mathbb{R}^{n}\right)$ is dense in $H^{p}\left(\mathbb{R}^{n}\right)$ (see [5]).

It is not difficult to see that we have discrete parameter versions of Theorems 1.1 and 2.4. To conclude this note we remark the following results.

Theorem 3.6. Let $\varphi$ be as in Theorem 1.1 and $0<p \leq 1$. Then, there exist positive constants $c_{1}, c_{2}$ such that

$$
c_{1}\|f\|_{H^{p}} \leq\left\|\left(\sum_{j=-\infty}^{\infty}\left|f * \varphi_{b^{j}}\right|^{2}\right)^{1 / 2}\right\|_{p} \leq c_{2}\|f\|_{H^{p}}
$$

for $f \in H^{p}\left(\mathbb{R}^{n}\right)$. 
Theorem 3.7. Let $0<p, q<\infty$ and $w \in A_{\infty}$. Suppose that $\varphi$ and $\psi$ fulfill the hypotheses of Theorem 2.4. Then we have, for $f \in \mathcal{S}\left(\mathbb{R}^{n}\right)$,

$$
\left\|\left(\sum_{j=-\infty}^{\infty}\left|f * \psi_{b^{j}}\right|^{q}\right)^{1 / q}\right\|_{p, w} \leq C\left\|\left(\sum_{j=-\infty}^{\infty}\left|f * \varphi_{b^{j}}\right|^{q}\right)^{1 / q}\right\|_{p, w} .
$$

\section{REFERENCES}

[1] A. Benedek, A. P. Calderón and R. Panzone, Convolution operators on Banach space valued functions, Proc. Nat. Acad. Sci. U. S. A. 48 (1962), 356-365.

[2] A. P. Calderón, Inequalities for the maximal function relative to a metric, Studia Math. $\mathbf{5 7}$ (1976), 297-306.

[3] A. P. Calderón, An atomic decomposition of distributions in parabolic $H^{p}$ spaces, Advances in Math. 25 (1977), 216-225.

[4] A. P. Calderón and A. Torchinsky, Parabolic maximal functions associated with a distribution, Advances in Math. 16 (1975), 1-64.

[5] A. P. Calderón and A. Torchinsky, Parabolic maximal functions associated with a distribution. II, Advances in Math. 24 (1977), 101-171.

[6] O. N. Capri, On an inequality in the theory of parabolic $H^{p}$ spaces, Revista de la Unión Matemática Argentina 32 (1985), 17-28.

[7] C. Fefferman and E. M. Stein, Some maximal inequalities, Amer. J. Math. 93 (1971), 107115.

[8] C. Fefferman and E. M. Stein, $H^{p}$ spaces of several variables, Acta Math. 129 (1972), 137193.

[9] G. B. Folland and E. M. Stein, Hardy Spaces on Homogeneous Groups, Princeton Univ. Press, Princeton, N.J. 1982.

[10] J. Garcia-Cuerva and J.L. Rubio de Francia, Weighted norm inequalities and related topics, North-Holland, Amsterdam, New York, Oxford, 1985.

[11] L. Grafakos, Modern Fourier Analysis, Third Edition, Graduate Texts in Math., no 250, Springer, New York, 2014.

[12] R. H. Latter and A. Uchiyama, The atomic decomposition for parabolic $H^{p}$ spaces, Trans. Amer. Math. Soc. 253 (1979), 391-398.

[13] J. Peetre, On spaces of Triebel-Lizorkin type, Ark. Mat. 13 (1975), 123-130.

[14] J. L. Rubio de Francia, F. J. Ruiz and J. L. Torrea, Calderón-Zygmund theory for operatorvalued kernels, Adv. in Math. 62 (1986), 7-48.

[15] S. Sato, Remarks on square functions in the Littlewood-Paley theory, Bull. Austral. Math. Soc. 58 (1998), 199-211.

[16] S. Sato, Vector valued inequalities and Littlewood-Paley operators on Hardy spaces, preprint (2015).

[17] E. M. Stein and G. Weiss, Fourier Analysis on Euclidean Spaces, Princeton Univ. Press, 1971.

[18] J. -O. Strömberg and A. Torchinsky, Weighted Hardy spaces, Lecture Notes in Math. 1381, Springer-Verlag, Berlin Heidelberg New York London Paris Tokyo Hong Kong, 1989.

[19] A. Uchiyama, Characterization of $H^{p}\left(\mathbb{R}^{n}\right)$ in terms of generalized Littlewood-Paley $g$ functions, Studia Math. 81 (1985), 135-158.

Department of Mathematics, Faculty of Education, Kanazawa University, Kanazawa 920-1192, JAPAN

E-mail address: shuichi@kenroku.kanazawa-u.ac.jp 